\title{
Intervenções não farmacológicas no manejo da dor do paciente adulto em terapia intensiva
}

\begin{abstract}
RESUMO
Objetivo: descrever o que as publicações cientificas trazem sobre o manejo da dor e medidas não farmacológicas utilizadas no cuidado ao paciente adulto internado em unidades de terapia intensiva. Metodologia: Foi realizado levantamento bibliográfico do período de 2008 a 2019 nas bases de dados da SciELO e BDENF. Utilizando os descritores: cuidados críticos, cuidados de enfermagem e manejo da dor. Utilizando os critérios de exclusão como cuidados ao neonatal, cuidados a parturiente, cuidados com o pé diabético, síndromes coronarianas. Resultado: A leitura dos artigos mostrou que os profissionais da área da saúde se limitam ainda a utilizar dos meios farmacológicos para trazer o alívio da dor nos pacientes, desconhecem também muitas técnicas que podem ser utilizadas para levar um conforto ao paciente. Conclusão: Ficou evidenciado a necessidade de os profissionais participarem de educações continuadas e da importância de um maior número de pesquisa nessa área.
\end{abstract}

DESCRITORES: Cuidados Críticos; Cuidados de Enfermagem; Manejo da Dor.

\section{ABSTRACT}

Objectives: describe what scientific publications bring about pain management and non-pharmacological measures used to care for adult patients admitted to intensive care units. Methodology: A bibliographic survey was conducted from 2008 to 2019 in the databases of SciELO and BDENF. Using keywords: critical patient, nursing care and pain management. Using exclusion criteria such as neonatal care, parturient care, diabetic foot care, coronary syndromes Result: Reading the articles showed that health professionals are still limited to using the media pharmacological to bring pain relief in patients; there are also many techniques that can be used to bring comfort to the patient. Conclusion: It was evidenced the need for professionals to participate in continuing education and the importance of a greater number of researches in this area.

KEYWORDS: Critical Care; Nursing Care; Pain Management.

\section{RESUMEN}

Objetivos: Describir lo que las publicaciones científicas aportan sobre el manejo del dolor y las medidas no farmacológicas utilizadas para atender a pacientes adultos ingresados en unidades de cuidados intensivos. Metodología: se realizó una encuesta bibliográfica de 2008 a 2019 en las bases de datos de SciELO y BDENF. Uso de las palabras clave: paciente crítico, cuidados de enfermería y manejo del dolor. Uso de criterios de exclusión como atención neonatal, atención de parto, cuidado del pie diabético, síndromes coronarios. Resultado: la lectura de los artículos mostró que los profesionales de la salud todavía están limitados a usar medios farmacológicos para aliviar el dolor en los pacientes, también desconocen muchas técnicas que pueden usarse para llevar comodidad al paciente. Conclusión: se destacó la necesidad de que los profesionales participen en la educación contínua y la importancia de una mayor investigación en esta área.

PALABRAS CLAVE: Cuidados Críticos; Cuidados de Enfermería; Manejo del Dolor.

RECEBIDO EM: 19/11/2019 APROVADO EM: 19/11/2019

\section{Wenderson Bruno Herculano da Silva}

Acadêmica do 6 período do Curso de Graduação em Enfermagem da Universidade Veiga de almeida.

\section{Eunice Maria Pereira Côrtes}

Acadêmica do 6 período do Curso de Graduação em Enfermagem da Universidade Veiga de almeida.

\section{Priscilla Oliveira da Silva}

Enfermeira. Professora Assistente do Curso de Graduação em Enfermagem da Universidade Veiga de Almeida. Doutoranda em Saúde Pública da Escola Nacional de Saúde Pública (ENSP/FIOCRUZ). 


\section{Michelle Amorim Ferreira \\ Acadêmica do 7 período do Curso de Graduação em Enfermagem da Universidade Veiga de almeida.}

\section{Paulo Roberto Ferreira Machado}

Enfermeiro, Especialização em MBA em Gestão em Saúde - UCAM; Especialização em Gestão em Saúde da Família - UERJ; Especialização em Desenvolvimento Gerencial de Unidades Básicas do Sistema Único de Saúde - FIOCRUZ, Professor Adjunto da Universidade Veiga de Almeida. Enfermeiro da Secretaria Municipal de Saúde - RJ.

\section{Viviane Reis Fontes da Silva}

Enfermeira. Mestre em Enfermagem, pelo Programa de Pós-Graduação em Saúde e Tecnologia no Espaço Hospitalar, pela Universidade Federal do Estado do Rio de Janeiro (UNIRIO). Vínculo institucional: Assessora I, na Coordenadoria Geral de Ciência, Tecnologia e Inovação em Saúde, da Secretaria Municipal de Saúde - Prefeitura Municipal do Rio de Janeiro.

\section{Cristiano Bertolossi Marta}

Enfermeiro. Pós-Doutor em Enfermagem. Professor Adjunto do Departamento de Fundamentos de Enfermagem da Faculdade de Enfermagem da Universidade do Estado do Rio de Janeiro. Coordenador Geral da Pós-Graduação de Enfermagem em Alta Complexidade da Universidade Veiga de Almeida. Diretor de Pesquisa da Universidade Veiga de Almeida. Pesquisador Líder do Núcleo de Avaliação de Tecnologias e Economia em Saúde e Segurança do Paciente (NATESSP) da Universidade Veiga de Almeida.

\section{INTRODUÇÃO}

A dor no paciente crítico é decorrente de algumas ações e atos realizados durante a internação na Unidade de Terapia Intensiva (UTI), incluindo as punções realizadas para coleta de exames laboratoriais, passagem de sondas, exames complementares, ou até mesmo por deixarmos de fazer algumas ações, como: troca de decúbito, deixando o paciente na mesma posição, às vezes desconfortáveis por longos períodos, não higienização correta; como, troca de fraldas ao constatar que o paciente está urinado ou evacuado, não aspiração das vias aéreas entre outras pequenas ações que podem facilitar para o conforto do paciente crítico ${ }^{(1,2)}$.

A equipe de enfermagem (enfermeiro e técnico em enfermagem) são os responsáveis por realizar a maior parte desses procedimentos invasivos, porém necessários para a recuperação desses pacientes durante sua internação hospitalar. Infelizmente, esses procedimentos são as principais fontes de dor, e o enfermeiro tem grande responsabilidade e detém de conhecimento cientificamente comprovado que pode agir na prevenção e diminuição da dor de seus pacientes. Essas ações devem ser priorizadas durante o cuidado ao paciente, assim como o aprimoramento e multiplicação do conhecimento técnico-científico, para a realização desses procedimentos ${ }^{(3,4)}$.
O esforço constante e intenso de todos os profissionais em salvar vidas em grave risco tem ocultado a percepção de que também é possível diminuir simultaneamente o seu sofrimento. Mesmo sabendo que uma importante parte da dor experimentada pelo paciente crítico pode ser prevenida ou aliviada, ainda se observa o pouco uso dos métodos de alívio e prevenção da dor nesses pacientes ${ }^{(5)}$.

A identificação das situações que possam ser estressantes ao paciente internado é importante, pois influência na tomada de decisões e no cuidado prestado pela equipe que o acompanha. Isso se torna um desafio, devido muitas vezes a impossibilidade de comunicação verbal entre os profissionais e os pacientes hospitalizados ${ }^{(1)}$. Um dos métodos é a avaliação adequada da dor, já que dela dependem as medidas resolutivas no controle e minimização do seu sofrimento, assim como a implementação de medidas não farmacológicas no seu cuidado diário, pois a avaliação da dor requer dos profissionais habilidades e conhecimento científico ${ }^{(3)}$.

Como alguns pacientes em estado crítico são incapazes de revelar a intensidade de sua dor, seja verbal ou não verbal, nessas situações, o enfermeiro deve utilizar outros indícios. Além de monitorar os parâmetros fisiológicos e sinais vitais, os prestadores do cuidado também podem prever e reconhecer as situações clínicas nas quais é provável que haja dor; podem ainda utilizar de seus conhecimentos de fisiologia e fisiopatologia e experiência com outros enfermos com problemas semelhantes. Ao combinar sua experiência com entrevista bem conduzida através de relatos da família, e a habilidade de observação, os enfermeiros de terapia intensiva podem avaliar a dor de seus pacientes de forma eficaz e intervir de modo adequado ${ }^{(2,3)}$.

Os sinais e sintomas da avaliação que frequentemente acompanham as reações de dor, são: aumento da frequência cardíaca, aumento da pressão arterial, aumento da frequência respiratória, dilatação da pupila, palidez e sudorese, náuseas e vômito ${ }^{(4,6,7)}$.

Embora os pacientes com dor aguda, muitas vezes, apresentem sinais e sintomas como esses, é importante salientar que a ausência de um ou todos esses sinais e sintomas não exclui a presença da dor. Normalmente, os pacientes gravemente enfermos expressão a dor de forma verbal e não verbal, essas expressões podem assumir diversas formas, algumas delas de maneira bem sutil que podem ser ignorados com facilidade, tais como: gemido, choro, grito, silêncio, caretas, estremecimento, imobilização, esfregar-se, balançar-se e agarrar o braço do enfermeiro ${ }^{(4,6,7)}$.

Embora tenham sido descritos correspondentes fisiológicos e comportamentais da dor aguda, a resposta de cada pessoa é única. Além disso, é importante lembrar 
que os pacientes que estejam recebendo agentes bloqueadores neuromusculares podem ser incapazes de apresentar sinais sutis de desconforto, até mesmo por causa da paralisia terapêutica. Esses bloqueadores neuromusculares não afetam os nervos sensitivos e não têm propriedade analgésica ${ }^{(7)}$.

Estratégias não farmacológicas são indicadas para a prevenção e diminuição da dor durante procedimentos relacionados com a dor aguda, de intensidades leve e moderada. Possuem eficácia comprovada e apresentam baixo risco para os pacientes, assim como baixo custo operacional concernente aos cuidados intensivos. Podem ser utilizados métodos, como: massagens, acupuntura, mudança de decúbito, aspiração adequada das vias aéreas, do tubo orotraqueal, arrumação do leito, troca de roupas, adequado posicionamento na cama hospitalar ou cadeira de rodas, luz adequada, som ambiente livre de ruídos, menor manipulação do paciente, promover conforto, distração, imaginação, termoterapia, musicoterapia, entre outros cuidados que podem ser implementados ${ }^{(2,6,8)}$.

Considerando que a terapia medicamentosa para alívio da dor pode apresentar alguns efeitos adversos, podendo comprometer o estado geral e futuro do paciente. A utilização de medidas não farmacológicas é benéfica e os estudos vêm comprovando sua eficácia. Assim, a revisão da literatura traz contribuições para a prática clínica do enfermeiro ao mostrar as evidências sobre o manejo da dor no paciente crítico, pois ao sistematizar esse conhecimento, ele servirá para orientar as práticas clínicas e intervenções que proporcionam uma melhora na vida do indivíduo sob o cuidado da enfermagem em UTIs ${ }^{(4,7)}$.

A pesquisa teve como questão: $\mathrm{O}$ que as evidências científicas mostram sobre as medidas não farmacológicas que o enfermeiro pode utilizar para diminuir a dor e aumentar o conforto do paciente adulto internado no Centro de Tratamento Intensivo?

O objetivo do estudo foi descrever o que as publicações cientificas trazem sobre o manejo da dor e medidas não farmacológicas utilizadas no cuidado ao paciente adulto internado em UTIs.
Esse estudo tem relevância de trazer para o debate as intervenções mais utilizadas em benefício desses clientes e minimizar o custo hospitalar, como também incentivar a uma educação continuada nos serviços de saúde concernente à manutenção, observação e intervenção da equipe de enfermagem no alívio da dor, diminuindo efeitos colaterais, distúrbios metabólicos, dependência medicamentosa e problemas renais que possam ocasionar pela utilização constante e ininterrupta de medicamentos ${ }^{(1,9)}$.

\section{METODOLOGIA}

Trata-se de uma revisão integrativa, método de pesquisa que sumariza resultados de pesquisa da literatura por meio de um processo de análise sistemática, seguindo as etapas: elaboração da questão de pesquisa, busca na literatura dos estudos primários com base nos critérios de inclusão e exclusão, organização dos estudos pré-selecionados, análises críticas dos estudos primários selecionados, análise e síntese dos resultados avaliados, apresentação da revisão integrativa ${ }^{(8)}$.

Figura 1. Fluxograma de seleção e leitura dos artigos. Rio de Janeiro, RJ, Brasil, 2019

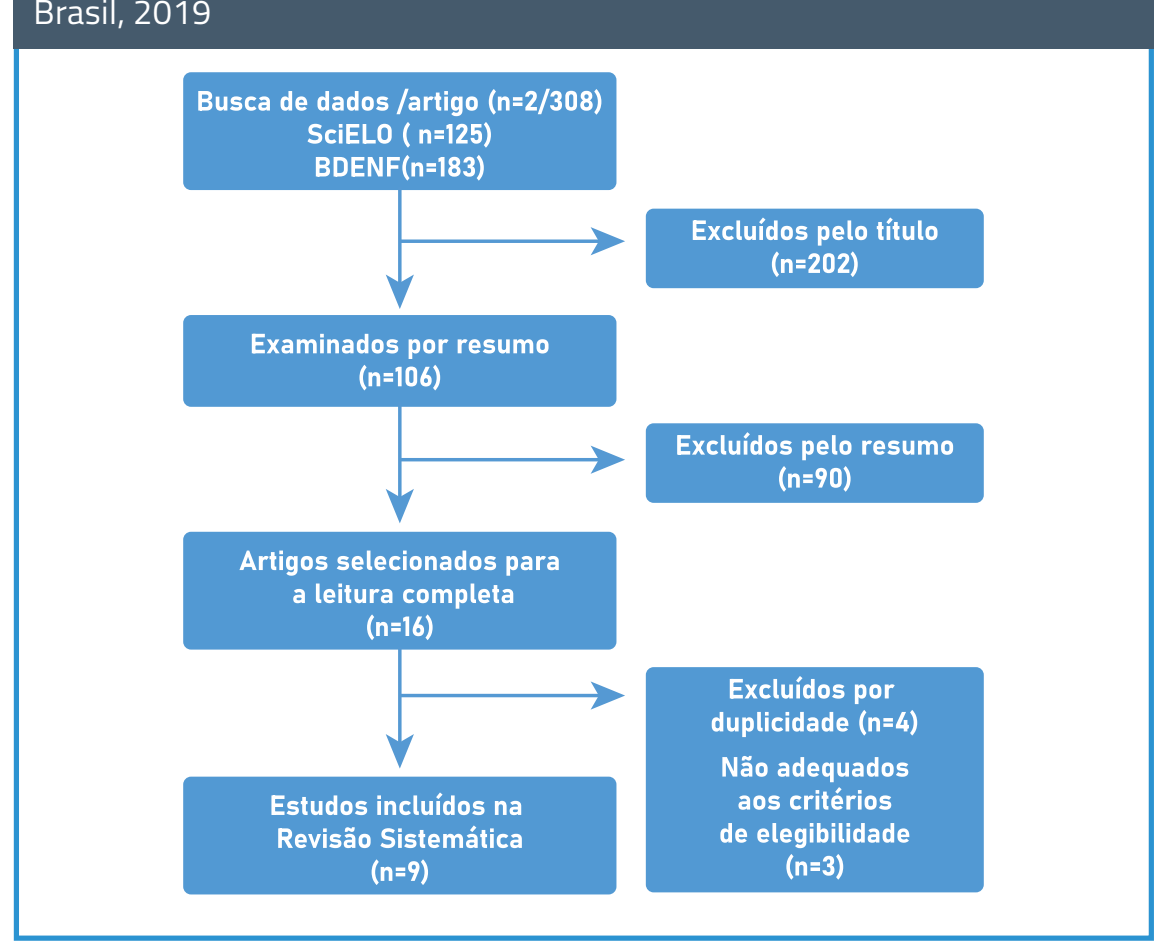

Os dados foram coletados no período entre julho a setembro de 2019 , as bases de dados utilizadas foram SciELO e Biblioteca Virtual de Saúde (BVS). Com os Descritores em Ciências da Saúde (DECS) "cuidados crítico", "cuidado de enfermagem" e "manejo da dor". Foram encontrados 125 artigos na SciELO e 183 artigos na BDENF, usando "OR" e "AND". Foram selecionados artigos na íntegra em português dos últimos 9 anos, devido ao pouco conteúdo encontrado nos últimos 5 anos sobre o tema. Seguiu-se com a leitura de 9 artigos encontrados com essa temática e dentro dos critérios estabelecidos de inclusão; os critérios de exclusão foram: teses e dissertações, editoriais e estudos de revisão, cuidado neonatal e à parturiente, cuidados com o pé diabético, síndromes coronarianas e grandes queimados.

Para avaliação crítica, realizou-se a leitura na íntegra dos estudos e a releitura dos resultados, com a finalidade de identificar aspectos relevantes que se repetiam ou se destacavam, esse fluxograma mostras como se seguiu para a escolha dos artigos de estudo. 


\section{artigo}

Silva, W.B.H.; Côrtes, E.M.P.; Silva, P.O.; Ferreira, M.A.; Machado, P.R.F.; Silva, V.R.F.; Marta, C.B.

Intervenções não farmacológicas no manejo da dor do paciente adulto em terapia intensiva

\section{RESULTADOS}

Os achados mostraram que a maior parte dos estudos sobre o tema ainda se concentram na avaliação da dor do paciente oncológico, este por ter uma maior visibilidade e muitos trabalhos e estudos de pesquisa voltados para os cuidados paliativos, possuem muito mais manejos e intervenções da enfermagem, juntamente com um olhar mais atento na prestação de cuidados dessas pessoas. No serviço prestado às outras clientelas que também se encontram internadas nas UTIs, as intervenções se restringem prioritariamente à administração medicamentosa seguindo as prescrições médicas. A monitorização e as intervenções de enfermagem ainda são incipientes, evidenciando fragilidades no manejo da dor.

As dificuldades encontradas para efetivar as intervenções no manejo da dor estão relacionadas, algumas vezes, aos próprios profissionais de saúde, seja por medo, insegurança, frustação, falta de empatia ou conhecimento científico; e ao cliente que, por muitas vezes ,não consegue verbalizar a dor ou o seu desconforto. A pesquisa também mostrou a deficiência das instituições em investir em uma educação continuada dos profissionais que prestam os cuidados aos pacientes internalizados, assim como uma alta demanda de serviço e, por muitas vezes, poucos profissionais que não conseguem atingir a demanda exigida, infraestrutura precária e desorganização do serviço ${ }^{(7)}$.

Observou-se também que a maioria dos artigos traz mais de uma medida não farmacológica que pode ser empregada no cuidado ao cliente que está necessitando de cuidados constantes, tais como: mudança de decúbito, aspiração adequada das vias aéreas, do tubo orotraqueal, arrumação do leito, troca de roupas, adequado posicionamento na cama hospitalar, luz adequada, som ambiente livre de ruídos, menor manipulação do paciente, promover conforto, distração, imaginação, termoterapia, musicoterapia e o próprio toque do profissional de saúde, fortalecimento do vínculo paciente/ profissional/família crioterapia $^{(2,6,9)}$.

Como resultado, também foi observado que os profissionais não fazem uso da escala para avaliação da dor, tanto da escala análogo numérica (EAN) que avalia a dor de 0 a 10 , sendo o zero sem dor e a 10 a pior dor imaginável, quanto da escala verbal descritiva, que varia entre nenhuma dor, leve, moderada e grave e a escala visual analógica - $\operatorname{EVA}^{(7,10)}$.

Com a leitura dos artigos foi feito um quadro com os achados mais importantes de cada publicação. Eles foram identificados por número, de acordo com a ordem de localização e organizado em fichas bibliográficas com os seguintes itens: título, autores, ano, base de dados, resultado e conclusão

Quadro 1. Extração dos dados dos artigos selecionados. Rio de Janeiro, RJ, Brasil, 2019

\begin{tabular}{|c|c|c|c|c|c|c|}
\hline ARTIGOS & TÍTULOS & AUTORES & ANO & BASE & RESULTADO & CONCLUSÃO \\
\hline 01 & $\begin{array}{l}\text { Conhecimento da equipe de } \\
\text { enfermagem sobre avaliação } \\
\text { comportamental de dor em } \\
\text { paciente crítico. }\end{array}$ & $\begin{array}{l}\text { Souza RCS, } \\
\text { Garcia DM, } \\
\text { Sanches MB, } \\
\text { Gallo AMA, } \\
\text { Martins VPF, } \\
\text { Siqueira } \\
\text { ILCP. }\end{array}$ & 2011 & SciELO & $\begin{array}{l}\text { Os resultados mostram as } \\
\text { barreiras que existem na } \\
\text { comunicação eficaz da dor, } \\
\text { como pouco conhecimento } \\
\text { e experiência, mostra } \\
\text { a importância de uma } \\
\text { capacitação adequada dos } \\
\text { profissionais. }\end{array}$ & $\begin{array}{l}\text { As equipes de } \\
\text { enfermagem conhecem } \\
\text { os pressupostos básicos } \\
\text { da dor, porém isso não } \\
\text { significa adesão integral } \\
\text { as ações adequadas ao } \\
\text { seu alívio. }\end{array}$ \\
\hline 02 & $\begin{array}{l}\text { Cuidados paliativos ao idoso } \\
\text { na terapia intensiva: Olhar da } \\
\text { equipe de enfermagem }\end{array}$ & $\begin{array}{l}\text { Queiroz TA, } \\
\text { Ribeiro ACM, } \\
\text { Guedes } \\
\text { MVC, } \\
\text { Coutinho } \\
\text { DTR, Freitas } \\
\text { MC. }\end{array}$ & 2018 & SciELO & $\begin{array}{l}\text { Alívio da dor e sofrimento, } \\
\text { manter conforto, Cuidar } \\
\text { na fragilidade, Carinho e } \\
\text { proteção, Higiene contínua, } \\
\text { Segurança do idoso, } \\
\text { Comunicação necessária, } \\
\text { Orientação para o cuidado. }\end{array}$ & $\begin{array}{l}\text { Falta de experiência no } \\
\text { cuidado, dificuldade da } \\
\text { equipe em mostrar-se } \\
\text { sensível as demandas da } \\
\text { familia. }\end{array}$ \\
\hline 03 & $\begin{array}{l}\text { Percepção dos profissionais } \\
\text { de enfermagem frente à } \\
\text { identificação, quantificação } \\
\text { e tratamento da dor em } \\
\text { pacientes de uma unidade de } \\
\text { terapia intensiva de trauma. }\end{array}$ & $\begin{array}{l}\text { Magalhães } \\
\text { PAP, Motta } \\
\text { FA, Saleh } \\
\text { CMR, Secco } \\
\text { LMD, Fusco } \\
\text { SRG, Gouvêa } \\
\text { AL. }\end{array}$ & 2011 & SciELO & $\begin{array}{l}\text { Os profissionais reconhecem } \\
\text { a dor como quinto sinal vital, } \\
\text { muitos ainda recorrem ao } \\
\text { médico para administração } \\
\text { farmacológica, muitos não } \\
\text { utilizam as escalas para } \\
\text { mensuração da dor. }\end{array}$ & $\begin{array}{l}\text { A maioria dos profissio- } \\
\text { nais de enfermagem tem } \\
\text { conhecimento insufi- } \\
\text { ciente sobre a identifi- } \\
\text { cação, quantificação e } \\
\text { tratamento da dor. }\end{array}$ \\
\hline
\end{tabular}




\begin{tabular}{|c|c|c|c|c|c|c|}
\hline 04 & $\begin{array}{l}\text { Padrão de interven- } \\
\text { ções de enfermagem } \\
\text { realizadas em vítimas de } \\
\text { trauma segundo o Nur- } \\
\text { singActivities Score. }\end{array}$ & $\begin{array}{l}\text { Nogueira } \\
\text { LS, Padilha } \\
\text { KG,Silva DV, } \\
\text { Lança EFC, } \\
\text { Oliveira EM, } \\
\text { Sousa RMC. }\end{array}$ & 2015 & SciELO & $\begin{array}{l}\text { Observa-se que com relação } \\
\text { às intervenções de enferma- } \\
\text { gem classificadas conforme } \\
\text { complexidade, os resultados } \\
\text { apontados mostram que } \\
\text { os paciente internados em } \\
\text { UTI necessitam de cuida- } \\
\text { dos além do normalmente } \\
\text { requeridos. }\end{array}$ & $\begin{array}{l}\text { Intervenções de enfermagem } \\
\text { analisadas: monitorização da } \\
\text { dor; medicação; procedimentos } \\
\text { de higiene; cuidados com dre- } \\
\text { nos; mobilização e posiciona- } \\
\text { mento; suporte e cuidado aos } \\
\text { familiares e pacientes; suporte } \\
\text { respiratório; cuidado com vias } \\
\text { aéreas artificiais. O artigo } \\
\text { também fala da importância } \\
\text { da capacitação da equipe no } \\
\text { cuidado. }\end{array}$ \\
\hline 05 & $\begin{array}{l}\text { Condutas terapêuticas } \\
\text { utilizadas no manejo da } \\
\text { dor em oncologia. }\end{array}$ & $\begin{array}{l}\text { Pereira DTS, } \\
\text { Andrade } \\
\text { LL, Agra G, } \\
\text { Costa MML. }\end{array}$ & 2015 & BDENF & $\begin{array}{l}\text { Esse artigo mostrou o uso } \\
\text { de massoterapia, banho de } \\
\text { imersão, crioterapia e banho } \\
\text { de aspersão quente, ainda } \\
\text { se faz muito comum o uso } \\
\text { apenas de fármacos. }\end{array}$ & $\begin{array}{l}\text { Diante do exposto, conclui- } \\
\text {-se que o controle da dor em } \\
\text { oncologia tem sido um desafio } \\
\text { para a prática clínica dos } \\
\text { profissionais de enfermagem, } \\
\text { Os resultados evidenciaram } \\
\text { predomínio das terapêuticas } \\
\text { farmacológicas, restringindo } \\
\text { o cuidado de enfermagem à } \\
\text { administração de medicamen- } \\
\text { tos. Quanto aos cuidados não } \\
\text { farmacológicos, constatou-se } \\
\text { que estes não são explorados } \\
\text { na sua plenitude, fato este } \\
\text { que pode ser justificado pelos } \\
\text { recursos materiais e humanos } \\
\text { insuficientes. }\end{array}$ \\
\hline 06 & $\begin{array}{l}\text { Conforto em cuidados } \\
\text { paliativos: O saber-fazer } \\
\text { do enfermeiro no hospital } \\
\text { geral. }\end{array}$ & $\begin{array}{l}\text { Durante } \\
\text { ALTC, Tonin } \\
\text { T, Armini LR. }\end{array}$ & 2014 & BDENF & $\begin{array}{l}\text { Cuidar envolve longos perí- } \\
\text { odos prestados ao doente } \\
\text { provocando desgaste físico } \\
\text { e psicológico, A comunicação } \\
\text { entre os profissionais de } \\
\text { saúde e as famílias é impres- } \\
\text { cindível e os enfermeiros são } \\
\text { os profissionais que exercem } \\
\text { papel fundamental na orien- } \\
\text { tação e treinamento desses } \\
\text { cuidadores/familiares. }\end{array}$ & $\begin{array}{l}\text { O saber-fazer do enfermeiro } \\
\text { sobre conforto em pacientes } \\
\text { em cuidados paliativos está } \\
\text { diretamente relacionado ao } \\
\text { seu componente físico, e en- } \\
\text { volve a realização de técnicas e } \\
\text { procedimentos para o alívio da } \\
\text { dor e sintomas exclusivamente } \\
\text { físicos. }\end{array}$ \\
\hline 07 & $\begin{array}{l}\text { Benefícios das práticas } \\
\text { integrativas e comple- } \\
\text { mentares no cuidado de } \\
\text { enfermagem. }\end{array}$ & $\begin{array}{l}\text { Mendes DS, } \\
\text { Moraes FS, } \\
\text { Lima GO, Sil- } \\
\text { va PR, Cunha } \\
\text { TA, Crosseti } \\
\text { MGO, Riegel } \\
\text { F. }\end{array}$ & 2019 & BDENF & $\begin{array}{l}\text { Aborda o uso da fitoterapia, } \\
\text { massagem, acupuntu- } \\
\text { ra, musicoterapia, toque } \\
\text { terapêutico, aromaterapia, } \\
\text { meditação, reiki, crioterapia, } \\
\text { hidroterapia }\end{array}$ & $\begin{array}{l}\text { A enfermagem possui papel } \\
\text { fundamental no que se refere } \\
\text { à aplicação dessas práticas } \\
\text { reconhecidas e respaldadas } \\
\text { pela legislação vigente. No } \\
\text { entanto é preciso preparo para } \\
\text { a identificação das necessida- } \\
\text { des dos pacientes, bem como } \\
\text { dos benefícios de cada tipo de } \\
\text { prática integrativa no cotidiano } \\
\text { do trabalho da enfermagem. }\end{array}$ \\
\hline
\end{tabular}




\begin{tabular}{|c|c|c|c|c|c|c|}
\hline 08 & $\begin{array}{l}\text { A enfermagem no } \\
\text { manejo da dor em } \\
\text { unidades de atendi- } \\
\text { mento de urgência e } \\
\text { emergência. }\end{array}$ & $\begin{array}{l}\text { Olivei- } \\
\text { ra PEP, } \\
\text { Pereira LV, } \\
\text { Santos NR, } \\
\text { Souza LAF. }\end{array}$ & 2016 & BDENF & $\begin{array}{l}\text { As intervenções para alívio } \\
\text { da dor ainda são prioritaria- } \\
\text { mente medicamentosas }\end{array}$ & $\begin{array}{l}\text { As barreiras apontadas para a efetiva- } \\
\text { ção do manejo da dor relacionam-se ao } \\
\text { próprio profissional (medo, insegurança, } \\
\text { frustração, falta de empatia com cliente, } \\
\text { entre outros), ao cliente (dificuldade em } \\
\text { entender as escalas utilizadas, insta- } \\
\text { bilidade clínica, relutância em aceitar } \\
\text { opioide) ou às questões organizacionais } \\
\text { da instituição, como deficiência nos pro- } \\
\text { cessos de educação continuada sobre } \\
\text { o tema, conflitos entre profissionais e } \\
\text { gestores, alta demanda de pacientes, } \\
\text { infraestrutura e organização do serviço }\end{array}$ \\
\hline 09 & $\begin{array}{l}\text { Treinamento e ava- } \\
\text { liação sistematizada } \\
\text { da dor: impacto no } \\
\text { controle da dor do pós- } \\
\text {-operatório de cirurgia } \\
\text { cardíaca }\end{array}$ & $\begin{array}{l}\text { Silva MAS, } \\
\text { Pimenta } \\
\text { CAM, Cruz } \\
\text { DALM. }\end{array}$ & 2013 & BDENF & $\begin{array}{l}\text { O estudo mostrou pouco } \\
\text { registro das intervenções de } \\
\text { enfermagem e do relato da } \\
\text { dor dos pacientes. Observa- } \\
\text {-se que na prática clínica em } \\
\text { UTI cirúrgica, principalmente } \\
\text { em pacientes de alto risco, } \\
\text { a equipe frequentemente } \\
\text { está mais preocupada com } \\
\text { o diagnóstico e o tratamen- } \\
\text { to das outras disfunções } \\
\text { orgânicas e pouco considera } \\
\text { a questão da dor. }\end{array}$ & $\begin{array}{l}\text { Aponta a necessidade de treinamento e } \\
\text { melhor anotações nos prontuários. }\end{array}$ \\
\hline
\end{tabular}

\section{DISCUSSÃO}

Observa-se com essa pesquisa de revisão integrativa que existe uma limitação na produção de trabalhos e pesquisas nessa área sobre a minimização da dor no paciente crítico. Quanto à escolha do recorte temporal, acredita-se que não teve interferência, uma vez que os estudos sobre medidas não farmacológicas para o alívio da dor são mais recentes.

Esse estudo contribui com o campo científico e prático, pois divulga sobre métodos não farmacológicos na redução e no alívio do sofrimento e estimula os profissionais a utilizar desses manejos, principalmente os enfermeiros para a aplicação dessas intervenções na sua rotina de trabalho. Além disso, incentiva a realização de outros estudos e pesquisas trazendo os benefícios dessas implementações terapêuticas no serviço de saúde.

Essa revisão integrativa contribui com a prática clínica da equipe de cuidados, realçando que a utilização não somente de fármacos para minimizar a dor é importante, pois a terapia medicamentosa apresenta muitos efeitos adversos em decorrência, algumas vezes, do estado clinico apresentado pelo paciente ${ }^{(7)}$.
Observou-se também que pela complexidade do quadro clinico, se faz necessária a interação de outros profissionais que também atendem ao necessitado desses cuidados, de uma equipe multiprofissional na avaliação do desconforto no paciente onde todos possam suprir e auxiliar na melhora do incomodo sofrido pelo hospitalizado. Artigos mostram que a enfermagem é consciente de que somente o próprio paciente pode mensurar a dor por ele sentida, isso sendo, muitas vezes, impossibilitado de acontecer devido ao estado apresentado. Isso requer do prestador de cuidados de enfermagem competência técnica, baseada no conhecimento adquirido através de estudos científicos para realizar esses cuidados de manejos e intervenções, associando objetividade com fatores subjetivos ${ }^{(2)}$.

Apontaram também que os profissionais de enfermagem compreendem a complexidade da experiência da dor e estão sensíveis a ela. Porém, algumas pesquisas mostram que os registros de dor feito por esses profissionais superestimam a porcentagem da dor referida pelo paciente, valoriza-se mais a questão física do que as psicossociais referidas pelo próprio ${ }^{(1)}$.

Artigos $^{(6,7)}$ citam que, nas clínicas UTIs, a equipe está frequentemente preocupada com o diagnóstico e tratamento das disfunções fisiológicas e poucos consideram a questão da dor, o que reforça a necessidade de lembrar aos profissionais de avaliar estes aspectos no doente e a necessidade de um protocolo a ser seguido. Sendo a dor um fator de estresse em terapia intensiva, alterando o padrão do sono e levando a uma exaustão, desorientação e agitação. A utilização pela equipe de enfermagem dos instrumentos para avaliar adequadamente a necessidade do paciente é essencial para o seu controle, sendo de extrema importância para a implementação do cuidado e qualidade no serviço prestado. Porém muitos desses profissionais têm dificuldade em utilizar essas escalas de avaliação, seja pelo desconhecimento ou pela dificuldade de interpretação dos pacientes. Esse estudo evidenciou as dificuldades apresentadas pelos técnicos também na medição da dor.

Pesquisas mostram algumas barreiras que devem ser consideradas no manejo da dor nas UTIs, incluindo: experiência do profissional, conhecimento científico adquirido, fatores relacionados ao paciente, ambientes encontrados, formas de comunicação, mudanças no estado clínico do internado, dis- 
ponibilidade ou não da tecnologia e a sua utilização, tempo reduzido no cuidado e quantitativo de profissionais da enfermagem para prestar assistência à uma quantidade elevada de pessoas necessitadas de cuidado ${ }^{(7)}$.

A avaliação da dor em um paciente crítico e impossibilitado de falar se torna crucial, visto que a dor é um componente vital para o seu tratamento e diagnóstico, podendo aumentar a morbidade e mortalidade da clientela. Capacitar a equipe que presta os cuidados é de vital importância para manter e melhorar a qualidade do cuidado, um cuidado inadequado acrescido da impossibilidade de comunicação do paciente referente à dor se constitui na dificuldade das intervenções da equipe ao paciente ${ }^{(4)}$.

Um estudo cita que a conduta dos enfermeiros em intervir em clientes com queixas álgicas foi significativamente melhorada após a participação da equipe em programas de educação continuada e capacitações. O manejo da dor pode ser alcançado através de desenvolvimento dos profissionais por meio de capacitação, treinamento e cursos oferecidos contínuos e estruturados ${ }^{(4,7)}$.

\section{CONCLUSÃO}

Com análise dos trabalhos pesquisados, salienta-se a importância de uma melhor monitorização da dor sofrida pelo paciente crítico para que a equipe de enfermagem saiba avaliar, monitorizar a dor, minimizar o sofrimento e intervir, até mesmo, preventivamente para diminuir os danos. Dessa forma, existe a necessidade de serem utilizadas escalas já criadas para esse fim, como já descritas anteriormente.

A pesquisa também mostrou que existe uma gama de trabalhos voltados para o cuidado ao paciente oncológico, observa-se que muitos desses artigos existem devido à especialização em cuidados paliativos, onde se busca trazer conforto e qualidade de vida ao paciente, já que não existe mais possibilidade de um tratamento curativo.

Sabe-se que existem barreiras que dificul- tam esses cuidados e manejos, descritos como a dificuldade da equipe em entender a escala de dor, impossibilidade de comunicação do paciente, distanciamento do enfermeiro do cuidado devido suas muitas atribuições burocráticas e à sobrecarga de trabalho dos profissionais envolvidos na arte do cuidar.

O que aponta para a necessidade de capacitar todos os prestadores de serviço vinculados a saúde a saber interpretar sinais e sintomas transmitidos pelos pacientes e capacitá-los a fazer os ajustes necessários para diminuir o desconforto do paciente. Essa necessidade pode ser suprida através das educações continuadas ministradas pelos órgãos prestadores de serviço. É importante salientar que a equipe de enfermagem é o profissional que está mais próximo ao paciente, porém não é o único responsável pelo cuidado, existe toda uma equipe multidisciplinar que está envolvida neste processo, desta forma, todos devem saber avaliar, monitorar e intervir em ações que minimizem a dor e o sofrimento e agilizem a recuperação do paciente.

\section{REFERÊNCIAS}

1. Souza RCS, Garcia DM, Sanches MB, Gallo AMA, Martins VPF, Siqueira ILCP. Conhecimento da equipe de enfermagem sobre avaliação comportamental de dor em paciente crítico. Revista Gaúcha de Enfermagem [Internet]. 2013 [acesso em 17 set 2019], 34:65:8. DOl: http://dx.doi.org/10.1590/s198314472013000300007.

2. Durante ALTC, Tonin T, Armini LR. Conforto em cuidados paliativos: $\mathbf{O}$ saber-fazer do enfermeiro no hospital geral. Revista de enfermagem UFPE on-line [Internet]. 2014 [acesso em 18 ago 2019]; 8:530:6. Disponivel em: https://periodicos. ufpe.br/revistas/revistaenfermagem/article/view/9707.

3. Queiroz TA, Ribeiro ACM, Guedes MVC, Coutinho DTR, Freitas MC. Cuidados paliativos ao idoso na terapia intensiva: olhar da equipe de enfermagem. Texto \& Contexto Enfermagem [Internet]. 2018 [acesso em 10 set 2019]; 27. Disponivel em:www.scielo.br/scielo.php?script=sci_arttext\&pid=S0104-07072018000100310\&Ing=p\%20t\&tIng=pt.

4. Magalhães PAP, Motta FA, Saleh CMR, Secco LMD, Fusco SRG, Gouvêa AL.Percepção dos profissionais de enfermagem frente à identificação, quantificação e tratamento da dor em pacientes de uma unidade de terapia intensiva de trauma. Rev. Dor [Internet]. 2011 [acesso em 10 set 2019]; 12(3):221224. Disponivel em: http://www.scielo.br/scielo.php?scrip$\mathrm{t}=\mathrm{sci}$ _arttext\&pid=S1806-00132011000300005\&Ing=p $\% 20$ t\&nrm=iso\&tlng=en\#nd.

5. Nogueira LS, Padilha KG, Silva DV, Lança EFC, Oliveira EM, Sousa RMC. Padrão de intervençõ̂es de enfermagem realizadas em vítimas de trauma segundo o Nursing Activities Score. Revista da Escola de Enfermagem da USP [Internet]. 2015 [acesso em 10 ago 2019]; 49(2):6-9. Disponível em:
http://www.scielo.br/pdf/reeusp/v49nspe/1980-220X-reeusp-49-spe-0029.pdf.

6.Pereira DTS, Andrade LL, Agra G, Costa MML. Condutas terapêuticas utilizadas no manejo da dor em oncologia. Revista online de pesquisa: Cuidado é fundamental [Internet]. 2015 [acesso em 10 set 2019]; 7(1):1883. Disponivel em: http://www.seer. unirio.br/index.php/cuidadofundamental/article/view/3578.

7.Oliveira PEP, Pereira LV, Santos NR, Souza LAF. A enfermagem no manejo da dor em unidades de atendimento de urgência e emergência. Revista Eletrônica de enfermagem [Internet]. 2016 [acesso em 27 set 2019]; 18(21):234. Disponivel em: https://revistas.ufg.br/fen/article/view/37309.

8. Mendes DS, Moraes FS, Lima GO, Silva PR, Cunha TA, Crosseti MGO, Riegel F. Benefícios das práticas integrativas e complementares no cuidado de enfermagem. Journal Health Npeps [Internet]. 2019 [acesso em 27 set 2019]; 302(16):1-4. Disponivel em: https://periodicos.unemat.br/ index.php/jhnpeps/article/view/3452.

9. Mendes KDS, Silveira RCCP, Galvão CM. Revisão integrativa: método de pesquisa para a incorporação para evidências na saúde e na enfermagem. Texto \& Contexto Enfermagem [Internet]. 2008 [acesso em 15 set 2019]; 17(4):758-756. DOI: http://dx.doi.org/10.1590/s0104-07072008000400018.

10. Silva MAS, Pimenta CAM, Cruz DALM. Treinamento e avaliação sistematizada da dor: impacto no controle da dor do pós-operatório de cirurgia cardíaca. Revista da Escola de Enfermagem da USP [Internet]. 2013 [acesso em 15 set 2019]; 47(1):84-88. DOl: http://dx.doi.org/10.1590/s008062342013000100011 . 